\title{
ADMINISTRAÇÃO DE MEDICAMENTOS: AQUISIÇÃO DE CONHECIMENTOS E HABILIDADES REQUERIDAS POR UM GRUPO DE ENFERMEIROS
}

Paulo Celso Prado Telles Filho ${ }^{2}$ Silvia Helena de Bortoli Cassiani ${ }^{3}$

Telles PCP Filho, Cassiani SHB. Administração de medicamentos: aquisição de conhecimentos e habilidades requeridas por um grupo de enfermeiros. Rev Latino-am Enfermagem 2004 maio-junho; 12(3):533-40.

Atualmente, sabe-se que o processo da administração de medicamentos é multidisciplinar e exige atualização constante. Escassez de conhecimento, falta de reciclagem e de educação em serviço permeiam a temática da administração de medicamentos. Objetivos: analisar as necessidades educacionais de enfermeiros atuantes em uma instituição hospitalar universitária do interior do Estado de São Paulo, no que concerne à administração de medicamentos e discutir sobre necessidade da aquisição de conhecimentos e habilidades requeridos pelo grupo de enfermeiros em questão. Utilizou-se da Técnica do Grupo Nominal por meio da seguinte questão: "O que você necessita saber mais para complementar seu conhecimento sobre a administração de medicamentos?" Destacaram-se oito categorias de necessidades educacionais: mecanismos de ação dos medicamentos, preparo e administração de medicamentos, obtenção de informações e conhecimento, interações medicamentosas, estabilidade do medicamento, medicamentos específicos e quimioterápicos, efeitos colaterais dos medicamentos e outros aspectos da administração de medicamentos. Propõem-se um curso de atualização, baseado nas necessidades educacionais identificadas.

DESCRITORES: preparações farmacêuticas; educação em enfermagem; enfermagem

\section{MEDICATION ADMINISTRATION: ACQUISITION OF KNOWLEDGE AND ABILITIES REQUIRED FOR A GROUP OF NURSES}

Nowadays, it is known that medication administration is a multidisciplinary process and requires constant updating from the professionals in charge. However, lack of knowledge, updating and in-service education permeate this theme area. Goals: analyze the educational needs of nurses working at a university hospital in the interior of São Paulo State, Brazil with regard to medication administration, as well as discuss about the need for this group of nurses to obtain the required knowledge and abilities. The Nominal Group Technique was used by means of the following question: "What else do you need to know in order to complete your knowledge on medication administration?" Eight categories of educational needs emerged: medication effect mechanisms, medication preparation and administration, information and knowledge acquisition, medication interactions, medication stability, specific and chemotherapeutic medication, side effects of medication and other aspects of medication administration. An updating course was proposed on the basis of the identified educational needs.

DESCRIPTORS: pharmaceutical preparations; nursing education; nursing

\section{ADMINISTRACIÓN DE MEDICAMENTOS: ADQUISICIÓN DE CONOCIMIENTOS Y HABILIDADES REQUERIDOS POR UN GRUPO DE ENFERMEROS}

Actualmente, es sabido que el proceso de administración de medicamentos es multidisciplinar y exige del profesional responsable actualización constante. Sin embargo, escasez de conocimiento, falta de reciclaje y de educación en servicio, están juntos a la temática de la administración de medicamentos. Objetivos: analizar las necesidades educacionales de enfermeros que actúan en una institución hospitalaria universitaria del interior del Estado de São Paulo, Brasil, con respecto a la administración de medicamentos, y también discutir acerca de la adquisición de conocimientos y habilidades requeridos por un grupo de enfermeros. Fue utilizada la Técnica del Grupo Nominal a través de la siguiente cuestión: "¿Lo qué más usted necesita saber para complementar su conocimiento sobre la administración de medicamentos?" Ocho categorías de necesidades educacionales se destacaron: mecanismos de acción de los medicamentos, preparo y administración de medicamentos, obtener de informaciones y conocimiento, interacciones medicamentosas, estabilidad del medicamento, medicamentos específicos y quimioterápicos, efectos colaterales de los medicamentos y otros aspectos de la administración de medicamentos. Los autores propusieron un curso de actualización, basado en las necesidades educacionales anteriormente identificadas.

DESCRIPTORES: preparaciones farmacéuticas; educación en enfermería; enfermería

\footnotetext{
${ }^{1}$ Trabalho extraído da Dissertação de Mestrado, defendida na Escola de Enfermagem de Ribeirão Preto, da Universidade de São Paulo em 21/ 12/2001; ${ }^{2}$ Enfermeiro, Mestre em Enfermagem Fundamental, Docente da Faculdade de Enfermagem da UNICASTELO, Campus VIII, e-mail: paulocptelles@bol.com.br; ${ }^{3}$ Enfermeira, Professor Associado da Escola de Enfermagem de Ribeirão Preto, da Universidade de São Paulo, Centro Colaborador da OMS para o desenvolvimento da pesquisa em enfermagem
} 
INTRODUÇÃO

$\boldsymbol{A}$ equipe de enfermagem é responsável pela administração dos medicamentos aos clientes em todas as instituições de saúde. Fato é que tal atividade revestese de grande importância para profissionais e clientes envolvidos, à medida em que é experiência cotidiana, de responsabilidade legal da equipe de enfermagem, e ocupa papel de destaque na função terapêutica a que o cliente está submetido.

Portanto, é imprescindível que a equipe de enfermagem, durante a terapêutica medicamentosa, observe e avalie sistematicamente o cliente quanto a possíveis incompatibilidades farmacológicas, reações indesejadas, bem como interações medicamentosas, com o intuito de minimizar riscos ao cliente. Salienta-se que ao enfermeiro cabe a detecção precoce, a prevenção de riscos e de possíveis complicações advindas da terapia medicamentosa $^{(1)}$. Destaca-se que tais observações e avaliações somente poderão ser oriundas de profissionais com conhecimento em farmacologia.

A esse respeito, há de se difundir e promover o conhecimento farmacológico aos profissionais para que, assim, incorporem uma base apropriada para a administração de medicamentos.

São também imprescindíveis os conhecimentos acerca de outras áreas, tais como anatomia, fisiologia, microbiologia e bioquímica, para a administração segura de medicamentos.

Administrar medicamentos é, portanto, um processo multi e inter-disciplinar, que exige do indivíduo, responsável pela administração, conhecimento variado, consistente e profundo. Por conseguinte, é fundamental, também, o conhecimento sobre os princípios que envolvem a administração de medicamentos, ação, interações e efeitos colaterais, uma vez que um erro, pode trazer graves conseqüências aos clientes sob responsabilidade desses profissionais $^{(2)}$.

Depara-se, por exemplo, com situações cotidianas em que os profissionais apresentam dificuldades quando da realização dos cálculos dos medicamentos pelo não domínio de questões matemáticas.

Tem-se, também, que o escasso conhecimento referente a métodos, vias, locais, ações, dosagens, diluições, técnicas, assepsia (inclusive a lavagem das mãos) e utilização adequada de materiais estéreis podem promover considerável parcela de erros no processo da administração de medicamentos.

Existem comentários acerca do binômio: falta de conhecimento e procedimentos falhos ${ }^{(3-4)}$. Verifica-se, por conseguinte, a estreita correlação entre conhecimento e a problemática dos erros na administração de medicamentos.

Na busca pela amenização dessa problemática, pesquisadores da área têm indicado a necessidade de capacitação do profissional que administra medicamentos como intervenção fundamental para a minimização de tais ocorrências.

Problemas relatados com a terapia de drogas podem ser evitados com a intervenção preventiva, dentre elas destaca-se a educação da equipe de enfermagem envolvida na administração de medicamentos como fator importante para a prevenção dos erros de medicação ${ }^{(5)}$.

Portanto, a prevenção da problemática descrita relaciona-se diretamente à educação e ao conhecimento.

Cumpre esclarecer que o presente estudo justificase à medida que, identificando e compreendendo as necessidades educacionais dos enfermeiros e propondo um curso que objetive minimizá-las ou saná-las, contribuase para uma administração de medicamentos segura, eficaz e com nível de excelência, o que proporcionará aos indivíduos que administram medicamentos e, principalmente, àqueles que os recebem, benefícios de incontestável valor.

\section{OBJETIVOS}

Analisar as necessidades educacionais de enfermeiros atuantes em uma instituição hospitalar universitária do interior do Estado de São Paulo, no que concerne à administração de medicamentos.

Discutir sobre a necessidade da aquisição de conhecimentos e habilidades requeridos pelos enfermeiros em questão.

\section{METODOLOGIA}

Trata-se de um levantamento de necessidades, estudo esse em que o pesquisador coleta dados para avaliar as necessidades de um grupo, comunidade ou organização ${ }^{(6)}$.

O estudo realizou-se em uma instituição hospitalar universitária localizada no interior do Estado de São Paulo.

Enquanto estrutura física, a instituição é composta por 403 leitos ativos, contando com 38 enfermarias, 17 departamentos médicos, 44 especialidades médicas, 22 
unidades de procedimentos especializados, 15 salas de cirurgia, 8 salas cirúrgicas ambulatoriais, 8 serviços de ambulatório e 5 serviços de diagnóstico.

O Serviço de Enfermagem Médico-Cirúrgica I (SEMC I), cenário dessa investigação, é dividido em dois blocos ( $\mathrm{C}$ e E) e compreende as áreas que se seguem: 2 salas administrativas e 4 enfermarias (sendo que cada enfermaria possui 36 leitos e é composta por posto de enfermagem, expurgo, sala de reuniões, copa para funcionários, copa de serviço de nutrição, sala de guarda de material, serviço de higiene e limpeza).

A seleção do referido serviço deveu-se à ocorrência do elevado volume e complexidade de medicamentos a serem administrados, bem como à existência de um serviço atuante de educação continuada na referida instituição.

A população foi constituída por 51 enfermeiros que compõem o SEMC I da instituição hospitalar universitária, ou seja, um enfermeiro diretor de serviço, oito supervisores e quarenta e dois enfermeiros assistenciais.

No entanto, foram excluídos o enfermeiro diretor de serviço e seis enfermeiros supervisores, que não se disponibilizaram a participar, um enfermeiro supervisor, que estava participando de um evento científico em outra localidade, seis enfermeiros assistenciais, que estavam em período de férias, dois enfermeiros, que não se disponibilizaram a participar, um, que estava de "folga", um, em licença prêmio, e uma, em licença gestante. Portanto, a amostra constituiu-se de 32 enfermeiros, sendo um enfermeiro supervisor e trinta e um enfermeiros assistenciais.

A decisão pela utilização da Técnica de Grupo Nominal, originalmente Nominal Group Technique, deveuse à possibilidade de ela atingir os objetivos propostos e visara maximizar a produtividade criativa do grupo, facilitar a decisão grupal, estimular a geração de idéias críticas, economizar esforço humano e auxiliar como instrumento no agrupamento de idéias.

Tal técnica constitui-se de cinco etapas, padronizadas da seguinte forma: $1^{\underline{a}}$ etapa: Elaboração escrita das respostas individuais; $2^{\underline{a}}$ etapa: Registro das respostas; $3^{\underline{a}}$ etapa: Discussão das respostas para esclarecimentos; 4⿳亠丷厂 Etapa: Voto preliminar das respostas e $5^{\mathrm{a}}$ etapa: Peso ${ }^{(7)}$, e foram percorridas no presente estudo.

Foram formados oito grupos nominais com os participantes do estudo, seguindo a recomendação da literatura que aponta nove indivíduos como sendo o máximo aceitável para cada grupo.

A composição desses grupos foi resultante da articulação entre pesquisador e diretor de serviço da instituição. Os horários foram previamente agendados, considerando-se, também, a disponibilidade dos participantes. Vale ressaltar que o pesquisador participou de todos os grupos e dirigiu-os.

O estudo foi iniciado após a aprovação do Comitê de Ética em Pesquisa do hospital investigado. Foi esclarecido aos participantes que os resultados da pesquisa destinar-se-iam à elaboração de trabalho científico e possível publicação, que seria garantido o sigilo e o anonimato dos entrevistados, que os pesquisadores comprometer-se-iam em prestar informações e esclarecimentos adicionais diante de quaisquer dúvidas a respeito da pesquisa, as quais, por ventura, pudessem ocorrer, e que a desistência de sua participação no trabalho de pesquisa poderia ocorrer quando considerassem conveniente, sem que isso acarretasse dano pessoal e/ ou profissional para si próprios ou para a instituição a qual estivessem vinculados.

Cada participante tomou conhecimento do termo de consentimento livre e esclarecido e, em concordando com a participação no estudo, emitiu registro escrito.

A questão nominal apresentada foi: "O que você necessita saber mais para complementar seu conhecimento sobre a administração de medicamentos?"

\section{RESULTADOS}

Obtiveram-se, inicialmente, respostas com significados semelhantes ou idênticos. Devido a tal fato e objetivando um melhor entendimento de seus significados, optou-se por reuni-las em categorias, conforme evidenciado na Tabela 1.

Tabela 1 - Distribuição, em ordem decrescente, das categorias de resposta segundo pontuação. CampinasSP, 2001

\begin{tabular}{lc}
\hline \multicolumn{1}{c}{ CATEGORIAS } & $\begin{array}{c}\text { PONTUAÇAO } \\
\text { TOTAL }\end{array}$ \\
\hline 1 - Mecanismos de ação dos medicamentos & 8.839 \\
2 - Preparo e administração de medicamentos & 6.180 \\
3 - Obtenção de informações e conhecimento & 3.701 \\
sobre o processo da administração de & \\
medicamentos & \\
4 - Interações medicamentosas & 3.416 \\
5 - Estabilidade dos medicamentos & 2.718 \\
6 - Medicamentos específicos e quimioterápicos & 2.302 \\
7 - Efeitos colaterais dos medicamentos & 1.944 \\
8 - Outros aspectos da administração de & 1.554 \\
medicamentos & \\
\hline
\end{tabular}


Salienta-se que tais categorias não foram previamente construídas, mas, sim, emergiram dos dados.

As respostas dos enfermeiros (códigos) foram agrupadas, e as categorias, nominadas de acordo com a literatura, por exemplo: para as repostas dos enfermeiros (códigos) mecanismos de ação - absorção e tempo de duração do efeito e metabolismo - sua relação com a meia vida, teve-se a categoria mecanismos de ação dos medicamentos.

A seguir, as denominações das categorias foram validadas por três juízes e consideradas adequadas.

Ora, as respectivas categorias apresentam-se em ordem decrescente de pontuação, e, após a citação de cada item, descrevem-se as respectivas respostas e aspectos abordados dos participantes de todos os grupos.

A categoria 1, mecanismos de ação dos medicamentos, conta com 8.839 pontos, portanto, foi a que mais se destacou. Reuniu as respostas referentes aos mecanismos de ação, à absorção e tempo de duração do efeito, ao metabolismo e sua relação com a meia vida, à biodisponibilidade, às vias de eliminação, à absorção, à atuação da droga em pacientes transplantados e à absorção do medicamento por meio de diferentes vias.

$\mathrm{Na}$ categoria 2, preparo e administração dos medicamentos, obtiveram-se 6.180 pontos, os quais foram reunidos por meio das respostas referentes à diluição, validade, temperatura, horário, tempo de infusão e segurança na dosagem dos medicamentos.

A Categoria 3, obtenção de informações e conhecimento sobre o processo da administração de medicamentos, conta com 3.701 pontos e aborda os seguintes aspectos: informação sobre patologias, indicações e contra-indicações, maior conhecimento acerca dos fármacos, relação de igualdade entre nome comercial e nome científico, descrição atualizada, farmacologia, indicações, atualização e princípios ativos.

Segue-se a categoria 4, interações medicamentosas, a qual obteve 3.416 pontos e reuniu duas respostas de grande pontuação e importância no que tange à segurança na administração de medicamentos: interações medicamentosas e compatibilidade $x$ associação de solutos.

$\mathrm{Na}$ Categoria 5, estabilidade dos medicamentos, obtiveram-se 2.718 pontos, os quais foram somados por meio de respostas relativas à estabilidade em temperatura ambiente e em geladeiras, fotossensibilidade relacionada à embalagem e ao tempo, estabilidade após a diluição e tempo de validade dos medicamentos após o preparo.

A Categoria 6, medicamentos específicos e quimioterápicos, apresentou 2.302 pontos, contou com dez respostas e tratou de variados aspectos referentes a medicamentos específicos e, em especial, aos quimioterápicos.

A Categoria 7, efeitos colaterais dos medicamentos, obteve 1.944 pontos e tratou dos seguintes aspectos: efeitos colaterais, processo alérgico e reações adversas.

Finaliza-se com a categoria 8, outros aspectos da administração de medicamentos, que contou com 1.554 e reuniu as respostas que assim se seguem: quem deve estar presente quando se administra medicação nova ou com potencial elevado para efeitos colaterais, a qual se trata de um item auto-explicativo, não somando comentário algum; iatrogenias relacionadas à administração de medicamentos, suscitando comentário sobre que atitudes tomar para não ser iatrogênico na administração de medicamentos; como medicar e avaliar a intensidade da dor, na qual se comentou sobre quando administrar "analgésicos - se necessário" e que parâmetro utilizar para avaliação da dor; vias de administração, em que se comentou acerca de quais seriam os melhores locais de aplicação para as diversas vias de administração de medicamentos e, também, em jejum, para cirurgias ou exames, quando administrar medicamentos e que volume não prejudica o jejum, também auto-explicativa e não fomentando comentário algum.

\section{DISCUSSÃo}

Em relação à categoria mecanismos de ação dos medicamentos, faz-se interessante destacar que os termos ação, efeito e resposta são usados como sinônimos e referem-se ao resultado da interação de uma droga com uma entidade biológica do corpo ${ }^{(8)}$.

Dessa forma, é de fundamental importância que os enfermeiros estejam atentos a tal categoria para promoverem ações educativas, pois é sabido que, atualmente, a administração de medicamentos exige 0 conhecimento acerca dos mecanismos de ação das drogas administradas aos clientes.

Em relação à absorção e tempo de duração do efeito das medicações, em quase todos os casos, as 
drogas devem atravessar as membranas plasmáticas para atingir seus locais de ação. A facilidade com que um composto atravessa as membranas é a chave para a avaliação das taxas e extensão da absorção e distribuição da droga pelos vários compartimentos do organismo ${ }^{(8)}$.

Portanto, faz-se necessário o estudo profundo desse aspecto da farmacologia para a compreensão do processo de absorção e duração do efeito dos medicamentos. Por meio desse estudo, o enfermeiro tornase capaz de realizar a administração de medicamentos com maior segurança, minimizando a ocorrência de erros de medicação e, conseqüentemente, de complicações em clientes, advindas da administração de medicamentos.

No que concerne à categoria dois, que trata do preparo e administração de medicamentos, especificamente no que diz respeito à diluição, sabe-se que diferentes drogas exigem distintos volumes de diluente e que se deve observar casos de extremos de idade, função renal prejudicada, alterações da pressão arterial e restrição hidroeletrolítica. Nesses casos, deve-se avaliar a redução do volume recomendado para a diluição $0^{(9)}$.

Essa avaliação, se eficazmente realizada, em muito facilita o processo de recuperação dos clientes, pois não permite que órgãos ou sistemas sejam ainda mais sobrecarregados.

Outra avaliação extremamente importante para a administração de medicamentos é a verificação da sua validade. Trata-se de um ato relativamente simples e que pode evitar um erro de medicação. No que tange à via de escolha e à temperatura em que o medicamento deve ser administrado, faz-se necessária a leitura acurada da prescrição médica, bem como da bula do medicamento em questão.

Em relação à categoria três, mais especificamente às informações sobre patologias, indicações e contraindicações dos medicamentos, o melhor meio de resolver dúvidas específicas relativas à pesquisa básica ou clínica é recorrer aos periódicos de farmacologia geral e especialidades clínicas ${ }^{(10)}$. Nos Estados Unidos da América, instituições divulgam boletins computadorizados que trazem notícias relativas a drogas recentemente aprovadas ou retiradas de circulação, e avisos em geral. No Brasil, tem-se como fontes de informação: bulas, compêndios de bulas e fichários informativos acerca dos medicamentos.

No entanto, ao estudar profissionais de enfermagem no que concerne à falha no conhecimento, foi detectado que, na maioria de sua amostra, houve registro de conhecimento deficiente em relação ao medicamento a ser administrado ${ }^{(1)}$. Tal resultado é extremamente contrário ao preconizado pelos estudiosos em administração de medicamentos, uma vez que o conhecimento acerca do fármaco que se administra é fundamental para a administração segura de medicamentos.

Em relação às informações atualizadas, no que concerne aos conhecimentos, práticas e em relação aos materiais utilizados para a administração de medicamentos, aponta-se para o fato de que $50 \%$ do conhecimento técnico em qualquer campo tem se tornado obsoleto em um prazo de cinco anos. Isso implica uma invasão crescente de novas tecnologias no cotidiano, fato que causa alterações na quantidade, qualidade e velocidade da troca de informação com que convivemos diariamente $^{(11)}$.

Em relação à categoria 4, faz-se importante destacar que a expressão interações medicamentosas refere-se a uma alteração na magnitude ou duração da resposta farmacológica a uma droga, devido à presença de outra droga ${ }^{(8)}$.

É imprescindível que a equipe de enfermagem, durante a terapêutica medicamentosa, observe e avalie o cliente quanto a possíveis interações medicamentosas e incompatibilidades farmacológicas, minimizando riscos a ele ${ }^{(1)}$.

Em relação à categoria cinco, que diz respeito à estabilidade dos medicamentos, destaca-se a necessidade de que se verifiquem as especificações do fabricante do medicamento quanto à necessidade de cuidados especiais, como a refrigeração, pois, para um mesmo princípio ativo, produzido por diferentes fabricantes, pode haver variações quanto à sua estabilidade.

A esse respeito, salienta que faz-se importante regular a temperatura da geladeira entre $4^{\circ}$ e $8^{\circ} \mathrm{C}$, reservando-a exclusivamente para o armazenamento de medicamentos, devendo ser descongelada e limpa periodicamente, abrindo-a o menos possível, por curtos períodos e freqüentemente verificar a vedação da porta. Esses cuidados, aparentemente simples, são importantes para se evitar um erro de medicação, ou seja, a administração de um medicamento que não está em condições de ser utilizado ${ }^{(9)}$.

Em relação à fotossensibilidade relacionada à 
embalagem dos medicamentos, cita-se que os medicamentos fotossensíveis apresentam-se acondicionados em embalagens especiais e que as instruções para o manuseio desses medicamentos devem ser rigorosamente seguidas.

$\mathrm{Na}$ categoria que trata acerca de medicamentos específicos e quimioterápicos, ressalta-se a atenção para o fato de que, ao profissional que atua nesta área, é requerido um conhecimento sobre biologia do câncer, farmacologia, princípios da quimioterapia do câncer, especificidade dos agentes antineoplásicos e os mais importantes princípios que governam a administração de quimioterápicos $^{(12)}$. O conhecimento desses itens é que destaca o enfermeiro dos outros membros da equipe da enfermagem, promovendo uma administração de medicamentos livre de imperícias.

O enfermeiro que busca a administração de medicamentos segura e tem conhecimento dos efeitos colaterais, pode estar agindo no sentido de minimizá-los tanto quanto possível.

Ressalta-se que, em todas as questões relacionadas a medicamentos específicos, recomenda-se a busca pelo conhecimento, ou seja, a obtenção de informações relativas a cada medicamento. Somente por meio de profundo estudo, será possível adquirir uma sólida base de conhecimento, o que, certamente, representará uma importante conquista para os enfermeiros e sua clientela.

No que concerne à categoria sete, ou seja, efeitos colaterais dos medicamentos, é relatado que os fármacos também ativam o sistema imune de modo indesejável, provocando manifestações alérgicas ${ }^{(13)}$. Essas reações manifestam-se sob a forma de erupções cutâneas, edema, reações anafilactóides, febre e eosinofilia.

O enfermeiro atento a esses sinais é o profissional responsável pela comunicação desse fato à equipe médica, que, provavelmente, suspenderá ou substituirá a medicação. Tal atitude também é possível e recomendada quando da observação de reações adversas.

Quanto à categoria que trata dos aspectos gerais da administração de medicamentos, destaca-se a questão da iatrogenia, à medida que, seguidas aos erros de medicação, as conseqüências para os clientes foram lesões do sistema nervoso central, reações de hipersensibilidade, amputação de membros, diminuição da acuidade visual e auditiva, aumento de dor e óbito do cliente $^{(14)}$.
Em relação às vias de administração dos medicamentos, sabe-se que esse item é dependente das características de efeito esperadas pela equipe médica, estando sujeitas às vias de administração, à prescrição medicamentosa.

É importante destacar que a administração de medicamentos é de competência do enfermeiro, e todas essas categorias apontam para o fato de que há necessidade de uma reflexão acerca da importância que tal profissional tem destinado a essa questão.

Este é o profissional que, por meio de sua formação, deve estar capacitado a exercer, com habilidade, a função de administrar medicamentos ${ }^{(15)}$.

Portanto, embora não sendo responsáveis pela prescrição dos medicamentos, devem conhecer todos os aspectos e fases envolvidas no processo, objetivando evitar erros e enganos, com prejuízos ao cliente ${ }^{(16)}$.

A administração de medicamentos requer atributos, tais como, compromisso ético, habilidade técnica e conhecimento científico.

A necessidade de educação profissional por meio de processos de educação continuada, cursos de reciclagem e treinamentos periódicos em administração de medicamentos deve ser enfatizada, pois, assim, os profissionais assumirão a parcela de responsabilidade que a profissão Ihes confere ${ }^{(17)}$.

Concorda-se que:

"A pesquisa tem um papel significativo na sociedade e, como tal, um compromisso social. No âmbito da enfermagem, uma profissão eminentemente prática e voltada para a saúde e qualidade de vida das pessoas, a finalidade da pesquisa não se resume em conhecer, compreender ou propor formas de atuação. Sua finalidade vai além. A produção teórica é apenas uma parte do processo, que se conclui na prática e na devolução dos resultados, tanto para a comunidade científica como para aquela onde foi desenvolvido o estudo"(18)

Assim, estabeleceu-se a necessidade de desenvolver uma ação educativa, objetivando atender às necessidades educacionais por meio da análise dos dados. Embasados na vivência da prática profissional hospitalar, julgou-se que a mais adequada ação educativa seria propor um curso de atualização, onde haveria a possibilidade de debates, discussões, indicação e estudo do material bibliográfico utilizado.

Por conseguinte, a finalidade de tal curso é a de atualizar conhecimentos acerca das dificuldades 
identificadas, relacionadas à administração de medicamentos, que foram apresentadas por enfermeiros da instituição hospitalar em questão.

Intitulado Curso de atualização: Administração de medicamentos e farmacologia, ele possui carga horária de 18 horas, distribuídas em quatro dias.

A população-alvo compõe-se de enfermeiros da instituição em estudo, tendo-se o intuito de estender tal curso a outros profissionais.

Como conteúdo programático, tem-se: mecanismos de ação dos medicamentos, preparo e administração de medicamentos, obtenção de informações e conhecimento sobre medicamentos e sua administração, interações medicamentosas, estabilidade dos medicamentos, informações sobre quimioterápicos e outros medicamentos específicos, efeitos colaterais dos medicamentos, iatrogenias, vias de eliminação, jejum e administração de medicamentos, medicação para dor, dentre outros.

Enquanto estratégias de ensino, serão utilizadas aulas expositivas dialogadas, seminários, leituras e discussão de textos relacionados à temática. Serão também utilizados transparências e slides.

Os critérios de avaliação serão baseados na avaliação formativa e somativa, ou seja, nas discussões em grupo, as quais deverão ser fundamentadas nas leituras propostas, seminários apresentados e em um questionário final, respectivamente.

\section{CONCLUSÃO}

É evidente que as exigências do mercado de trabalho, no que diz respeito à administração de medicamentos, são crescentes e de consenso entre pesquisadores e profissionais envolvidos com a prática da enfermagem que o conhecimento ocupa papel de destaque entre essas exigências.

A situação retratada neste estudo, que teve a pretensão de trazer à luz as necessidades educacionais de uma amostra de enfermeiros atuantes em uma instituição hospitalar, revelou a necessidade de conhecimento acerca de importantes conteúdos relativos à administração de medicamentos. São eles: Mecanismos de ação dos medicamentos, preparo e administração de medicamentos, obtenção de informações e conhecimento, interações medicamentosas, estabilidade dos medicamentos, medicamentos específicos e quimioterápicos, efeitos colaterais dos medicamentos e diversos outros aspectos da administração de medicamentos.

Os dados parecem apontar que o enfermeiro pode estar administrando medicamentos com déficit de conhecimento em questões essenciais para a administração livre de erros.

Refletindo acerca dessas questões e ciente de que, em decorrência dessa escassez de conhecimento, ocorrem muitos erros e, também, de que pouco é realizado no sentido de verificar as causas desses erros por parte das instituições de saúde, já que as intervenções adotadas pelas chefias de enfermagem são de ordem punitiva e não educativa, houve a proposição do curso, conforme explicado anteriormente.

No entanto, sabe-se que somente um curso não é suficiente para a erradicação dos erros de medicação.

Nesse cenário, em que a necessidade de investimento no processo educativo é emergencial, destaca-se a educação continuada, a qual indica-se para sanar a problemática retratada nesse estudo.

Assim se define educação continuada ${ }^{(19)}$ :

"Processo prolongado que vai para além dos limites dos sistemas educacionais, fazendo-se presente por toda a vida dos indivíduos, situados em uma sociedade em contínuas transformações; essa sociedade produz novas tecnologias, novos conhecimentos, mobilizando as possibilidades e os saberes dos profissionais, colocando a necessidade de continuidade na sua formação".

Tem-se, portanto, que a educação continuada dos profissionais da equipe de enfermagem é, atualmente, considerada como fator indispensável para a qualidade da prestação do serviço de enfermagem, já que ela pressupõe o entendimento e vivência do papel do enfermeiro enquanto educador, enquanto indivíduo que favorece e propicia situações de aprendizado, com o objetivo de crescimento contínuo e de tornar-se mais atuante, favorecendo a qualidade do serviço.

Recomenda-se, portanto, um efetivo programa de educação continuada, com treinamentos periódicos, incentivo à realização de estudos sobre temáticas diversas relacionadas à administração de medicamentos, inclusive em relação aos erros de medicação, investigando as causas e propondo soluções, promoção de conteúdos atualizados à equipe de enfermagem, desenvolvimento de pesquisas e consultas freqüentes a outros profissionais da saúde e à literatura.

Assumir a responsabilidade de qualificação 
periódica e atualizada em relação à administração de medicamentos é atitude obrigatória do enfermeiro, no sentido de prevenir danos e potencializar benefícios.

Não é a mera reflexão sobre a prática o que the introduz um fator crítico, senão a ação reflexiva, isto é, aquela que é a materialização da consciência transformadora ${ }^{(20)}$.

\section{REFERÊNCIAS BIBLIOGRÁFICAS}

1. Carvalho V T. Erros na administração de medicamentos: análise de relatos dos profissionais de enfermagem. [Dissertação]. Ribeirão Preto (SP): Escola de Enfermagem de Ribeirão Preto/USP; 2000.

2. Puschel VAA. Apresentação. In: Enfermagem: cálculo e administração de medicamentos. São Paulo (SP): Legnar Informática \& Editora; 1999. p.11-3.

3. Madlon-Kay DJ, Mosch FS. Liquid medication dosing errors. J Farm Pract 2000 Aug; 49(8):741-4.

4. Cassiani SHB, Rangel SM, Tiago F. Complicações após aplicações, por via intramuscular, do diclofenaco de sódio: estudo de um caso. Medicina 1998 jan/mar; 3(1):99-105.

5. Van Den Bemt PM, Egberts TA, Jong-Berg LT, Brouwers JR. Drug-related problems in hospitalized patients. Drug Saf 2000 Apr; 22(4):321-33.

6. Polit DF, Hungler BP. Fundamentos de pesquisa em enfermagem. 3a ed. Porto Alegre (RS): Artes Médicas; 1995. 7. Delbecq AL. Van de Vem AH, Gustafson D H. Group techniques for program planning: a guide to nominal group and delphi processes. Green Biar; 1986.

8. Brody TM, Larner J, Minneman KP, Neu HC. Farmacologia humana: da molecular à clínica. $2^{\underline{a}}$ ed. Rio de Janeiro (RJ): Guanabara Koogan; 1997.

9. Fakih FT. Manual de diluição e administração de medicamentos injetáveis. Rio de Janeiro (RJ): Reichmann \& Afonso; 2000.

10. Katzung BG. Farmacologia básica \& clínica. 6ª ed. Rio de Janeiro (RJ): Guanabara Koogan; 1998.

11. Shapiro J, Hughes S. Information technology as a liberal art. Educ Rev 1996; 31(1):31-5.

12. Weinstein S. Plumer's principles and practices of intravenous therapy. 5aㅡ. ed. Philadelphia: Lippincott; 1993.

13. Barbuto JAM, Hersh EM, Salmon SE. Imunofarmacologia. In Katzung BG, Silva P. Farmacologia básica \& clínica. Rio de Janeiro (RJ): Guanabara Koogan; 1995. p. 656-71.

14. Wolf ZR, Mcgoldrik TB, Flynn ER, Warnick F. Factors associated with a perceived harmful outcome from medication errors: a pilot study. J Contin Educ Nurs 1996; 27(2):65-74.

15. Miasso Al. Terapêutica Medicamentosa: orientação e conhecimento do paciente na alta e pós-alta hospitalar. [dissertação]. Ribeirão Preto (SP): Escola de Enfermagem de Ribeirão Preto/USP; 2002.

16. Carvalho VT, Cassiani SHB, Chiericato C, Miasso AI. Erros mais comuns e fatores de risco na administração de medicamentos em Unidades Básicas de Saúde. Rev Latinoam Enfermagem 1999; 7(5):67-75.
Transformar a prática da administração de medicamentos é papel de destaque do enfermeiro. Esperase que o presente estudo inicie essa transformação, contribuindo para a administração de medicamentos livre de erros, o que é uma importante fatia da promoção da qualidade de assistência aos diversos clientes, sob a responsabilidade do enfermeiro.

17. Carvalho VT, Cassiani SHB. Erros na medicação e conseqüências para profissionais de enfermagem e clientes: um estudo exploratório. Rev Latino-am Enfermagem 2002 julho-agosto; 10(4):523-9.

18. Hense DSS, Elkert ER, Penna CMM. A devolução dos resultados de pesquisa: uma questão de cidadania. Texto Contexto Enfermagen 1994 jan/jun; 3(1):92-101.

19. Bagnato MHS, Cocco MIM, De Sordi MRL. Educação, saúde e trabalho: antigos problemas, novos contextos, outros olhares. Campinas (SP): Alínea; 1999.

20. Lhavador FB. Prologo à la edición española. In: Usher R, Bryan I. La educación de adultos como teoria practica e investigación: el triángulo cautivo. Madrid: Morata; 1992. 